\title{
Resiliencia y discapacidad de los estudiantes de la carrera de Trabajo Social de la Universidad Técnica de Manabí
}

\author{
Resilience and Disability of the students of the Social Work Career of the Technical University of \\ Manabí.
}

\author{
Tatiana Katiuska Moreira Chica ${ }^{1}$ (D) (8), Evelyn Michelle Vélez García 2 (D) (8), \\ Sandra Auxiliadora Romero Chávez ${ }^{3}$ (iD (8)
}

\begin{abstract}
Cómo citar
Moreira et al. (2021). Resiliencia y Discapacidad de los estudiantes de la Carrera de Trabajo Social de la Universidad Técnica de Manabí. Socialium, 5(2), 297-317. https://doi.org/10.26490/uncp.sl.2021.5.2.934
\end{abstract}

${ }^{1}$ Magister en Educación y Desarrollo Social,

Licenciada en Trabajo Social, Universidad Técnica de Manabí,

Portoviejo, Ecuador. tatiana.moreira@utm.edu.ec

2 Egresada de la Carrera de Trabajo Social, Universidad Técnica de Manabí, Portoviejo, Ecuador. evelez9475@utm.edu.ec

3 Magister en Educación y Desarrollo Social, Licenciada en Trabajo Social, Universidad Técnica de Manabí, Portoviejo, Ecuador.

sandra.romero@utm.edu.ec

Arbitrado por pares ciegos Recibido: 04/05/2021 Aceptado: 29/06/2021

\section{RESUMEN}

La resiliencia en los estudiantes con discapacidad es de suma importancia, puesto que les permite afrontar las adversidades en el proceso de formación en la educación superior, por lo que requieren de entornos positivos que motiven y contribuyan al logro de sus metas. El objetivo de este proceso investigativo consistió en determinar la resiliencia y tipo de discapacidad de los estudiantes de la Carrera de Trabajo Social de la Universidad Técnica de Manabí. El desarrollo de la investigación fue de tipo no experimental, el cual se apoyó en el método analítico, estadístico, descriptivo y bibliográfico; así mismo se aplicó el método lógico científico: inductivo-deductivo, con enfoque cuali-cuantitativo; se utilizó la técnica de la encuesta y como instrumento la ficha de encuesta, considerando las fuentes interactivas de la resiliencia y las dimensiones de la escala validada de SV-RES, la misma que fue aplicada a 30 estudiantes con discapacidad de la Carrera de Trabajo Social de la UTM. Los resultados evidenciaron que los estudiantes sujetos de estudio de la Carrera de Trabajo Social tienen diferentes tipos de discapacidad y su resiliencia para afrontar adversidades es una capacidad desarrollada, debido a que la mayoría afirma poseer factores resilientes "yo soy, yo tengo y yo puedo" considerando las dimensiones de la escala validada de SV-RES, para lo cual cuentan con el apoyo de personas que constituyen su entorno como la familia, amigos, compañeros, docentes, Unidad de inclusión, Universidad Técnica de Manabí y Programas de bienestar del Estado ecuatoriano.

Palabras clave: resiliencia; discapacidad; inclusión; educación superior.

\section{ABSTRACT}

Resilience in students with disabilities is of utmost importance, since it allows them to face adversities in the process of training in higher education, so they require positive environments that motivate and contribute to the achievement of their goals. The objective of this research process was to determine the resilience and type of disability of the students of the Social Work Career of the Technical University of Manabí. The development of the research was nonexperimental, which was supported by the analytical, statistical, descriptive and bibliographic method; likewise, the logical-scientific method was applied: inductive-deductive, with a qualitative-quantitative approach; the survey technique was used and the survey form was used as an instrument, considering the interactive sources of resilience and the dimensions of the validated SV-RES scale, which was applied to 30 students with disabilities of the Social Work Career of the UTM. The results showed that the students under study of the Social Work Career have different types of disabilities and their resilience to face adversities is a developed capacity, since most of them claim to have resilient factors "I am, I have and I can" considering the dimensions of the validated SV-RES scale, for which they have the support of people who constitute their environment such as family, friends, classmates, teachers, Inclusion Unit, Technical University of Manabi and Welfare Programs of the Ecuadorian State.

Keywords: resilience, disability, inclusion, higher education. 


\section{Introducción}

La resiliencia es un término muy utilizado en la actualidad por muchos profesionales que interactúan con personas que presentan situaciones problemáticas, pues la misma tiene que ver con las capacidades que tiene el individuo para afrontar dificultades, fomentando técnicas que permitan fortalecerla y con ello, elevar la calidad de vida de los involucrados. Connor y Davidson, son citados por (Serrano et al., 2021, p. $582)$, quienes ponen de manifiesto que "La resiliencia es la capacidad para afrontar y superar situaciones de adversidad permitiendo a la persona un funcionamiento saludable".

La resiliencia presenta diversas definiciones que no permiten llegar a un acuerdo para poder de manera clara y concisa, determinar su alcance y dimensiones puesto que, haciendo referencia a situaciones adversas, hay personas que estando en extrema pobreza y vulnerabilidad social han logrado cosechar triunfos y mejores condiciones de vida. "La resiliencia se refiere a los comportamientos que despliega el ser humano frente a condiciones adversas que derivan en una adaptación exitosa" (Salvo et al., 2021, p. 3).

Es entonces la resiliencia una capacidad para adaptarse a situaciones del entorno, que de una u otra forma afecta el normal desarrollo normal de una persona, si se hace referencia al ser humano y si se trata de un elemento material es la capacidad para volver a su estado natural.

Es la resiliencia entonces una capacidad que desarrolla el ser humano, obligado en muchas ocasiones por las circunstancias adversas que lo rodean, a sobrellevar las mismas o a convivir con ellas teniendo al mismo tiempo una vuela calidad de vida. "La resiliencia puede ser apreciada como el resultado de una armonía entre factores de riesgo, factores protectores y la personalidad del ser humano. La persona puede estar más que resiliente" (Romero et al., 2018, p. 71).

Es normal que cualquier ser humano en algún momento de su vida haya tenido que enfrentar una situación adversa, algunos se han resignado a dejarse llevar por las consecuencias nefastas de la misma y otros han optado por enfrentarlas, a tal punto que logran desarrollar un sinnúmero de actividades y en muchas ocasiones con niveles altos de dificultad, tal es el caso de las personas con discapacidad, que con falencias y limitaciones afrontan situaciones muy complejas y negativas.

Las personas con discapacidad son aquellas que presentan algún tipo de deficiencia, ya sea de tipo motriz, física, intelectual o sensorial, situación que en varias ocasiones no les permite llevar una vida plena y efectiva en la sociedad, por lo que se han convertido en seres de lucha para que las políticas, normas o leyes que los amparan se ejecuten en todos los ámbitos donde se desenvuelven. Se considera a una persona con discapacidad según el Reglamento de la Ley Orgánica de Discapacidades, art. 1 (2017): 
La persona con discapacidad, para efectos de este Reglamento y en concordancia con lo establecido en la Ley, se entenderá por persona con discapacidad a aquella que, como consecuencia de una o más deficiencias físicas, mentales, intelectuales o sensoriales, con independencia de la causa que la hubiera originado, ve restringida permanentemente su capacidad biológica, psicológica y asociativa para ejercer una o más actividades esenciales de la vida diaria, en una proporción equivalente al treinta por ciento (30 \%) de discapacidad, debidamente calificada por la autoridad sanitaria nacional.

Durante mucho tiempo se ha mantenido en desigualdad y aislamiento a este grupo de atención prioritaria; sin embargo, con el transcurrir del tiempo la lucha por el reconocimiento de sus derechos y la inclusión en todos los ámbitos ha tenido resultados satisfactorios, los mismos que están establecidos leyes y decretos a nivel mundial.

Uno de los logros alcanzados en beneficio de las personas con discapacidad es el reconocimiento del derecho a la educación, integrándolos a dicho proceso y que los docentes apliquen metodologías incluyentes considerando sus necesidades educativas especiales, tal como se menciona en la Ley Orgánica de Discapacidades, art. 27 y 28 (2012), Sección III. De la educación:

Derecho a la educación, el Estado procurará que las personas con discapacidad puedan acceder, permanecer y culminar, dentro del Sistema Nacional de Educación y del Sistema de Educación Superior, sus estudios, para obtener educación, formación y/o capacitación, asistiendo a clases en un establecimiento educativo especializado o en un establecimiento de educación escolarizada, según el caso.

Educación inclusiva. - La autoridad educativa nacional implementará las medidas pertinentes, para promover la inclusión de estudiantes con necesidades educativas especiales que requieran apoyos técnico-tecnológicos y humanos, tales como personal especializado, temporales o permanentes y/o adaptaciones curriculares y de accesibilidad física, comunicacional y espacios de aprendizaje, en un establecimiento de educación escolarizada.

La educación es un derecho básico reconocido a nivel mundial, que ofrece garantías a todos y en especial a las personas con discapacidad que a pesar de las dificultades que enfrentan también poseen capacidades para afrontar las adversidades debido a su situación, convirtiéndose así en estudiantes resilientes.

Con base a los antecedentes abordados en esta investigación se hace referencia a lo relevante de la misma, cuyo objetivo fue determinar la resiliencia y tipo de discapacidad de los estudiantes de la Carrera de Trabajo Social de la Universidad Técnica de Manabí para lo cual se llevó a cabo un tipo de estudio no experimental, apoyándose en el método analítico, estadístico, descriptivo y bibliográfico, el método 
lógico científico: inductivo-deductivo, con enfoque cuali-cuantitativo mixto, aplicando la técnica de la encuesta y la entrevista, los cuales permitieron obtener resultados que se detallan en este documento.

\section{Resiliencia y educación superior}

La resiliencia en la actualidad es un término muy utilizado debido a las diferentes situaciones que afectan al ser humano, así (Tarazona et al. 2008, p.12) manifiestan que la resiliencia se remite a "la capacidad de los individuos para afrontar la adversidad. Al respecto se destacan como cualidades el optimismo, el espíritu aventurero, el coraje, la auto comprensión, el humor, la capacidad de trabajar duro y la habilidad para relacionarse con otro".

Es, por tanto, la resiliencia, esa capacidad la que permite al ser humano continuar con la cotidianidad de sus vidas pese a las situaciones que lo afligen, "la resiliencia es la capacidad de una persona o un grupo para seguir proyectándose en el futuro a pesar de situaciones desestabilizadoras, de condiciones de vida difíciles y de traumas graves" (Ormaza et al., p. 132).

El mundo se desarrolla cada día a pasos acelerados y con ello el ser humano, el mismo que debe utilizar herramientas, recursos y la predisposición para estar a la par, puesto que la sociedad impone estándares que todos desean alcanzar, pues para esto en necesario plantearse metas y a través de un proceso de formación desarrollar un perfil basado en conocimientos y competencias. Una de las fortalezas de las personas resilientes es plantearse objetivos y lograrlos, tal es el caso de las personas con discapacidad que acceden a los centros de educación superior para alcanzar un título que les permita ejercer como profesionales a pesar de las adversidades que tengan que enfrentar en el camino.

La educación superior es una faceta por la que un estudiante con deseos de ser un profesional y elevar su calidad de vida son el motor para incursionar en la misma, pero ésta requiere un conjunto de acciones, que si lo desarrolla con interés y responsabilidad logrará sin duda el éxito deseado. El contexto universitario también requiere de componentes y estándares que le permitan al estudiante continuar con dichos procesos, pues implica tener un presupuesto para movilización, material de oficina, alimentación, internet, equipos tecnológicos, entre otros, que unidos a la fuerza de voluntad también necesitan el apoyo económico y moral de la familia, de no ser así el estudiante se verá obligado a trabajar y con ello la posibilidad de tener que abandonar los estudios debido a lo agobiante que resultaría, por lo que éstos deben estar preparados, desarrollando capacidades para evitar abandono y retraso en la formación académica.

Por tal razón Zambrano et al. (2018) mencionan que:

La capacidad de resiliencia en un estudiante universitario, es algo que debe ser diagnosticado desde el proceso de nivelación para la inserción en una carrera profesional, esto garantizará que 
éstos puedan superarse y enriquecerse a pesar de las dificultades de ordenes comunicativas, socioeconómicas, intelectuales, raciales y de género que se pueden presentar en el proceso formativo. (p. 44)

Así pues, hay muchas razones que impiden en muchas ocasiones, cumplir con la meta propuesta al estudiantado en la educación superior, no obstante, también se desarrollan capacidades para afrontar las adversidades y complicaciones que se pueden presentar en el transcurso de la misma.

Sin lugar a dudas, también existen estudiantes resilientes que enfrentan situaciones adversas y logran su objetivo con miras a obtener una mejor calidad de vida, ejemplo de ello son aquellos que tienen algún tipo de discapacidad, que enfrentado complicaciones y limitantes obtienen su título profesional.

Los estudiantes resilientes con discapacidad son personas que han experimentado rechazo, aislamiento, indiferencia y olvido que, sin duda, estas situaciones han llegado a desbordar a la persona, no sólo por el shock traumático, sino por los procesos de adaptación y cambios a una nueva forma de vida que debe experimentar tanto el afectado como para los que le rodean. Los procesos de transición de las personas con discapacidad inician desde la niñez, es por esto que Martínez (2018) afirma:

La transición de la niñez a la adolescencia y posteriormente a la adultez, pasa por unas fases de adaptación en las que, de manera general, desde edades muy tempranas se aprende a convivir con esta situación, y por tanto a desarrollar los aspectos resilientes desde prácticamente el inicio de la infancia. No obstante, hay ocasiones en el que el momento vital en el que se detecta o sobreviene la discapacidad es a lo largo de la vida, como por ejemplo sucede al tener una lesión medular como consecuencia de un accidente de tráfico, una enfermedad degenerativa, etc. ( $p$. 77)

La vida universitaria para los estudiantes con discapacidad es un espacio que requiere de la resiliencia para lograr establecer vínculos positivos, reafirmar los valores y evitar el aislamiento social que conduce a la discriminación y en otros casos hasta a la violencia, por lo que la resiliencia promueve conductas basadas en la solidaridad y cooperación que contribuyan al desarrollo de la sociedad.

Para el logro de una verdadera inclusión, los docentes juegan un papel importante, para lo cual deben estar previamente capacitados en cuanto al uso y aplicación de metodologías inclusivas que son la base fundamental para la resiliencia, puesto que dicho proceso hará sentir integrado e incluido al estudiante con discapacidad y al mismo tiempo desarrollar conocimientos, habilidades y destrezas de acuerdo a su condición.

Los docentes fomentan la capacidad resiliente de los estudiantes a través de un ambiente agradable, motivándolos a través del humor, la fantasía, el afecto, la aceptación de sí mismo, la ilusión, la alegría, el 
amor, la generosidad, el optimismo, la esperanza, lo que permite abrir al educador y el educando a nuevas experiencias en un contexto de seguridad, teniendo en cuenta sus límites.

Otro aspecto fundamental para el estudiante con discapacidad es el apoyo y acompañamiento de la familia, puesto que como núcleo central de la sociedad es generadora de resiliencia, lo que contribuye al desarrollo de destrezas y el logro de los objetivos de ambos, que a lo posterior será un triunfo familiar y social.

\section{Medición de las dimensiones de la Resiliencia}

Para poder medir el nivel de resiliencia varios autores han postulado instrumentos de acuerdo a la característica y particularidad de la situación a investigar, una de las más aplicadas en la actualidad es "la Escala de resiliencia SV-RES, la misma que La prueba SV-RES ofrece una real posibilidad de ser utilizada en la investigación e intervención psicoeducativa, focalizando la acción y permitiendo evaluar con mayor precisión el impacto" (Saavedra y Villalta, 2008, p. 38).

Se consideran doce factores de resiliencia que describen la interacción del sujeto consigo mismo, los otros y sus posibilidades, lo que brinda un perfil del sujeto, tal como lo mencionan Moscoso y Castañeda (2018), quienes hacen referencia a doce factores de resiliencia que describen la interacción del sujeto consigo mismo, los otros y sus posibilidades, lo que brinda un perfil del sujeto y éstas son:

\section{Las que responden al yo soy/yo estoy}

a. La "identidad" se refiere a los juicios generales a la que opta el individuo para interpretar hechos y acciones de modo estable a lo largo de su vida, que lo define y constituye como parte de su caracterización personal.

b. La "autonomía" comprende al vínculo que el sujeto establece consigo mismo para definir su aporte al entorno sociocultural.

c. La "satisfacción" describe los juicios que tiene la forma particular en una situación problemática.

d. "Pragmatismo" indica el sentido práctico para evaluar y enfrentar los problemas y orientar las acciones que realiza.

\section{Mientras tanto, las que responden al "yo tengo"}

a. "Vínculos" se refiere al valor de la socialización y redes sociales con raíces en la historia personal como relaciones vinculares, apego, sistema de creencias, entre otros, que sirven de base para la formación de la personalidad. 
b. "Redes" se define como los vínculos afecticos que establece la persona con su entorno social cercano y constituyen un apoyo para el sujeto.

c. "Modelos" hace referencia a que el sujete busca en sus redes sociales cercanas una guía para enfrentar sus problemas y superarlos. También se toman en cuenta las experiencias anteriores que sirven de referente frente a la resolución de distintas problemáticas.

d. "Metas" son los juicios que refieren al valor contextual de metas y objetivos definidos, dirigidos hacia un fin.

\section{En cuanto al "yo puedo"}

a. La "afectividad" es el auto-reconocimiento de la vida emocional del sujeto y se basa en las posibilidades sobre sí mismo y el vínculo con el entorno (tono emocional, humor, empatía).

b. La "autoeficacia" define las posibilidades de éxito que la persona reconoce en sí mismo como los límites, control de impulsos, responsabilidad ante los actos y manejo de estrés ante una situación problemática.

c. El "aprendizaje" indica poder rescatar la posibilidad de aprender y el crecimiento frente a una situación problemática. De esta manera, se aprovecha la experiencia vivida, se aprende de los errores y se evalúa el actuar.

d. La "generatividad" se refiere a la posibilidad de pedir ayuda a otros, crear y construir respuestas alternativas y planificar las acciones. (p. 168)

\section{Discapacidad}

Al hacer referencia a una persona con discapacidad existen varias definiciones, una de las más relevantes que es determinada por el Consejo Nacional para la igualdad de Discapacidades, menciona que ésta “involucra a aquellos que tengan deficiencias físicas, mentales, intelectuales o sensoriales a largo plazo y que, al coexistir con distintas barreras, puedan imposibilitar su inclusión plena y efectiva en la sociedad, en igualdad de condiciones con los demás" (CONADIS, 2008, p. 5).

La discapacidad afecta a muchas personas en el mundo, sin embargo, no hay barreras ni limitantes que impidan su inclusión, pues la sociedad les ha abierto la puerta para que incursionen en su entorno fomentando su autorrealización. La Organización Mundial de la Salud (OMS, 2011) dio a conocer que:

Más de mil millones de personas viven en todo el mundo con alguna forma de discapacidad; de ellas, casi 200 millones experimentan dificultades considerables en su funcionamiento. En los años futuros, la discapacidad será un motivo de preocupación aún mayor, pues su prevalencia está aumentando. Ello se debe a que la población está envejeciendo y el riesgo de discapacidad 
es superior entre los adultos mayores, y también al aumento mundial de enfermedades crónicas tales como la diabetes, las enfermedades cardiovasculares, el cáncer y los trastornos de la salud mental. (p. 5)

\section{Tipos de Discapacidad y sus Características}

Para evaluar el tipo y porcentaje de discapacidad que tienen las personas que presentan esta situación existe un equipo calificado en Medicina, Psicología y Trabajo social, a través de la aplicación de varios parámetros establecidos. EI CONADIS (2008) para este fin, emite una clasificación validada por el Sistema Único de Calificación de Discapacidad en el Ecuador, las cuales son:

- Discapacidad motriz: Se define como un daño, resultado de una imposibilidad que impide el desempeño motor del individuo afectado. Muchas de las veces las causas de esta discapacidad se relacionan a problemas ocurridos en la etapa gestante, a causas de que el bebé nace prematuro o a complicaciones durante el nacimiento. También pueden ser resultado de alguna lesión medular a causa de accidentes ocurridos o problemas en el organismo.

- Discapacidad física: Se refiere a deficiencias corporales y/o viscerales. Las primeras pueden ser evidentes como amputaciones de miembros superiores o inferiores, paraplejía o hemiparesia (falta de movilidad de la mitad del cuerpo). Las segundas pueden implicar un daño en los órganos internos y por lo tanto ser imperceptibles, como afectaciones a pulmones o corazón, fibrosis quística de páncreas, insuficiencia renal crónica terminal, entre otras. Estas enfermedades generan dificultades para caminar, subir y bajar gradas, controlar esfínteres, mantener el equilibrio, etc.

- Discapacidad sensorial: Incluye a los individuos con problemas de la vista y audición, los cuales dificultan la comunicación con la comunidad y su entorno, lo que genera una desvinculación del medio y disminución de la participación social. Las discapacidades visuales las padecen las personas que presentan ceguera o baja visión. En los dos casos se refiere a un alto grado de pérdida de visión, es decir que no ven absolutamente aún con el uso de lentes. Mientras que la discapacidad se refiere a personas con sordera bilateral total y/o sordera severa de ambos oídos. Puede presentarse por causas genéticas, congénitas, infecciosas, ocupacionales, traumáticas, tóxicas, envejecimiento, entre otras.

- Discapacidad intelectual: Una persona con discapacidad intelectual tiene dificultad para comprender ideas complejas, razonar, resolver problemas, tomar decisiones y desenvolverse en la vida diaria, lo cual influye en sus relaciones interpersonales y les convierte en personas fácilmente influenciables; dentro de esta categoría se encuentra el retraso mental en sus grados fronterizo, leve, moderado, grave y profundo. 
- Discapacidad psicosocial (mental): Esta discapacidad es causada generalmente por enfermedades como la esquizofrenia, trastorno bipolar, psicosis, entre otras. Se manifiesta a través de deficiencias o trastornos de la conciencia, comportamiento, razonamiento, estados de ánimo, afectividad, y/o comprensión de la realidad (irritabilidad, depresión, inestabilidad emocional crónica). (pp. 11-12)

Determinar la forma de discapacidad de un individuo es lo principal que se debe de realizar a temprana edad, para que por medio de los profesionales especializados se pueda establecer cuál es el porcentaje de discapacidad y tener una aproximación a las necesidades que requieren ser atendidas y así acogerse a los diferente programas y servicios que brinda las instituciones y ministerios de bienestar social que garanticen una mejor calidad de vida.

\section{Discapacidad y educación superior}

Las personas que tienen algún tipo de discapacidad están amparadas por leyes y normativas de inclusión en todos los ámbitos para el logro de su promoción social y autodesarrollo. Al hacer referencia a los estudiantes con discapacidad en las instituciones de educación superior, están siendo incluidos en todos sus aspectos, dando así cumplimiento a lo dispuesto.

La Universidad Técnica de Manabí (UTM, 2021) es una institución de educación superior cuyo objetivo es formar integralmente al ser humano para que contribuya al desarrollo del país, al logro de la justicia social, fortaleciendo la identidad nacional en el contexto pluricultural del país, la afirmación de la democracia, la paz, los derechos de las personas y las comunidades, la integración latinoamericana y mundial, así como la defensa y protección del medio ambiente. Como institución pionera de la inclusión de los estudiantes con discapacidad, ejecuta un modelo inclusivo que destaca la universalidad, que es el reconocimiento de la dignidad humana, lo cual incluye satisfacer las necesidades básicas a fin de erradicar progresivamente toda forma de discriminación y eliminar las barreras que no les permiten desarrollar sus habilidades y destrezas como personas.

El modelo planteado permite abordar el entorno en el que se encuentran los estudiantes con discapacidad, considerando los componentes de la comunidad universitaria, los programas sociales y a la familia, entes fundamentales en su proceso de adaptación y desarrollo, así como también el procedimiento para que pueda acceder a los servicios o beneficios de acuerdo con su discapacidad y así garantizar un buen estilo de vida físico, social y emocional de los involucrados.

El Departamento de Inclusión, Equidad Social y Género (DIESG) de la (UTM, 2012) adopta medidas de acción en el diseño de políticas internas que respalden a este grupo de personas ante eventuales situaciones de desigualdad, en este sentido, se han llevado acciones coordinadas con las autoridades pertinentes, para lo cual se ha realizado adaptaciones incluyentes en sus instalaciones, para la 
accesibilidad a personas con discapacidad (escaleras, rampas, ascensores, entre otros), lo cual permite facilitar el acceso a determinadas áreas.

La (UTM, 2019, p .3) mediante el Reglamento de Políticas de Acción Afirmativa respalda "la inclusión educativa de estudiantes con discapacidad y cuestiona totalmente cualquier tipo de discriminación, razón por la que busca promover el acceso, la inclusión, la permanencia y la culminación de Carreras en Instituciones de Educación Superior de personas con discapacidad" es el objetivo tanto de la Secretaría de Educación Superior, Ciencia, Tecnología e Innovación (Senescyt, 2018) como del CONADIS.

El convenio tiene como propósito desarrollar, coordinar y motivar acciones conjuntas entre ambas instituciones a fin de asegurar condiciones de igualdad y libertad para las personas con discapacidad en el Ecuador. De esta manera, el CONADIS se compromete a asesorar a las universidades sobre la Normativa Nacional INEN, la accesibilidad al medio físico, la comunicación y la atención pre-hospitalaria y de desastres y la Senescyt a dar cumplimiento a la misma a través de la capacitación y aplicación de dichas Normativas implicando a estudiantes, docentes, directivos y personal administrativo.

En este contexto la UTM ha dado un giro positivo hacia la inclusión construyendo facilidades estructurales acordes a las necesidades de quienes padecen discapacidades físicas, generando conciencia sobre el beneficio de un trato igualitario a hombres y mujeres, y en estos casos formando al personal para que tenga los conocimientos y destrezas necesarias para que les permitan atender de la mejor manera a los estudiantes con discapacidad.

\section{Acceso a la educación de estudiantes con discapacidad}

El Estado ecuatoriano dando cumplimiento a las disposiciones legales, establece políticas inclusivas que permitan acceder a la educación, sin distinción de raza etnia o condición física, reduciendo de esta manera la exclusión y priorizando el principio al buen vivir, garantizando el debido derecho para el acceso a la educación superior. La Constitución de la Republica del Ecuador,Art. 26 y 28 (2008) determina que: La educación es un derecho de las personas a lo largo de su vida y un deber ineludible e inexcusable del Estado. Constituye un área prioritaria de la política pública y de la inversión estatal, garantía de la igualdad e inclusión social y condición indispensable para el buen vivir. Las personas, las familias y la sociedad tienen el derecho y la responsabilidad de participar en el proceso educativo.

El Estado adoptará a favor de las personas con discapacidad medidas que aseguren: La inclusión social, mediante planes y programas estatales y privados coordinados, que fomenten su participación política, social, cultural, educativa y económica. (pp. 15; 22)

El gobierno del Ecuador a través del Acuerdo de la Secretaría de Acceso a la Educación Superior Ciencia, Tecnología e Innovación (Senescyt, 2018, p. 22) desarrolla políticas de acción afirmativa como "Mérito 
Territorial y Política de Cuotas con lo que favorecen el ingreso a universidades, escuelas politécnicas e institutos técnicos y tecnológicos de grupos vulnerables, entre los que se encuentran las personas con discapacidad".

Para garantizan el acceso a la educación superior a quienes, por su situación socioeconómica, discapacidad, etnia, entre otros, se toma en cuenta el índice de vulnerabilidad, en donde la Senescyt utilizará los datos que se ingresan en la encuesta de factores asociados para que éstos accedan a los programas que les ingresar, permanecer y terminar con éxito las carreras elegidas por los beneficiarios y La Universidad Técnica de Manabí, en estricto cumplimiento de la ley, es una de las pioneras a nivel nacional, que ha desarrollado este plan piloto, generando resultados positivos, puesto que en la actualidad ha formado muchos profesionales con discapacidad y algunos están ya ejerciendo la profesión.

\section{Método}

Tipo de estudio. Para la realización del proceso de investigación se utilizó un diseño no experimental de carácter descriptivo de corte transversal, con enfoque cuantitativo, a nivel empírico se utilizó la técnica de la encuesta estructurada para la recopilación de datos, el cuestionario de preguntas cerradas con la escala Likert, considerando la escala de Resiliencia SV-RES de los autores (Saavedra y Villalta 2008). La unidad de análisis estuvo caracterizada por estudiantes con discapacidad de la Carrera de Trabajo Social de la Universidad Técnica de Manabí.

En cuanto al enfoque cualitativo, se aplicó una entrevista estructurada a la Directora del Departamento de Inclusión y Equidad social de la Universidad Técnica de Manabí, para identificar los objetivos, fines, propuestas y acciones que se desarrollan en beneficio de los involucrados. Para el desarrollo de la investigación bibliográfica se accedió a fuentes seguras y confiables de Google Académico y libros, estudiando y rescatando información relacionada con las variables estudiadas.

Población y muestra. La población objeto de la investigación fue de 30 estudiantes con discapacidad, siendo una cantidad mínima y contando con los contactos para localizarlos, se trabajó con el total de la población, es decir los 30 estudiantes con discapacidad matriculados de primer a octavo nivel de la Carrera de Trabajo Social de la Universidad Técnica de Manabí, de los cuales el 53 \% tiene discapacidad sensorial y el 47 \% física, a los mismos se los contactó utilizando datos informativos proporcionados por la Unidad de Inclusión y Equidad Social, para lo cual se usó la herramienta virtual Google forms para que respondan cada una de las interrogantes. Para encuestar a los estudiantes que tienen discapacidad sensorial se solicitó el acompañamiento de la intérprete de lenguaje de señas que labora en el Departamento de Inclusión, Equidad y Género de la UTM, así como también de sus familiares. 


\section{Instrumentos de recolección de datos}

Cuestionario: Se elaboró un cuestionario en relación a los objetivos planteados en el estudio, para lo cual las autoras plantearon 5 preguntas incluyendo variables de la investigación, las mismas que son: 1) Género (masculino y femenino) Tipo de discapacidad (sensorial, física, intelectual, motriz) ; 2) Fuente interactiva de la resiliencia: "Yo soy" (Una persona fuerte, Capaz de controlar mi vida, Competente para alcanzar metas, Orgulloso de mis logros, Seguro de mí mismo) ; Fuente interactiva de la resiliencia: "Yo tengo" (Confianza y optimismo, Facilidad para establecer contacto con nuevas personas, Amigos / familiares que me apoyan, Compañeros que me apoyan en las actividades académicas, Docentes que aplican metodología inclusiva); Fuente interactiva de la resiliencia: "Yo puedo" (Cumplir tareas asignadas, Planear actividades en base a metas, Trabajar en equipo, Organizar mi tiempo y ser puntual, Motivar a otros). Las preguntas son cerradas, para lo cual se le solicitó a los 30 encuestados (estudiantes con discapacidad), elegir entre las alternativas de si/no.

Guía de entrevista: Se elaboraron 4 preguntas abiertas basadas en las variables estudiadas, las mismas que son: 1) ¿Cuáles con las actividades que está desarrollando actualmente el Departamento de Inclusión y Equidad Social de Género con el fin de fomentar la inclusión?; 2) ¿Cómo departamento de Inclusión social qué propuesta están prestos a ejecutar?; 3) ¿Cuáles con las actividades que está desarrollando actualmente el Departamento de Inclusión y Equidad Social de Género con el fin de fomentar la inclusión?; 4) ¿Considera usted que los estudiantes con discapacidad de la Carrera de Trabajo social son resilientes?. Las preguntas abiertas permiten obtener información más amplia de la temática investigada puesto que motivan a la o el entrevistado a dar su retroalimentación, usando sus propias palabras, sin restringir sus pensamientos.

Procedimiento de la recolección de datos. La aplicación de la encuesta se la realizó a 30 estudiantes con discapacidad de la Carrera de Trabajo social de la Universidad Técnica de Manabí, para lo cual se contactó a los mismos a través de WhatsApp, debido a que es una aplicación muy utilizada en la actualidad por su fácil manejo. Considerando la discapacidad de cada uno de los encuestados se contó con el apoyo de los familiares y la docente intérprete, a quienes se les proporcionó el enlace para que accedan a la herramienta virtual Google Forms y contestar cada una de las alternativas planteadas en la encuesta, lo cual se lo realizó en el transcurso de 5 días.

De igual forma se localizó a la Directora del Departamento de Inclusión, Equidad social de la Universidad Técnica de Manabí a través de llamada telefónica, debido a las restricciones de aislamiento por la pandemia del COVID-19, para posteriormente a través de video llamada realizarle la entrevista, apoyándose en las preguntas abiertas planteadas, la cual tuvo una duración de 28 minutos. 
Aspectos éticos. La información proporcionada por los estudiantes con discapacidad fue procesada y analizada cuidadosamente por las investigadoras, considerando el carácter confidencial que se requiere para este tipo de investigación, cuya información es utilizada con fines investigativos y académicos, guardando la reserva de la identidad de los encuestados, priorizando su condición de vulnerabilidad debido a la discapacidad de cada uno de ellos.

Análisis de los datos. Para realizar el procesamiento de los datos obtenidos en la encuesta aplicada se utilizó el Software Microsoft Excel, así mismo se realizó el análisis de los resultados obtenidos basándose en el método estadístico y descriptivo de todas las variables a través de (tablas contingencias). Se observó el resultado de las variables estudiadas que aparecen en la tabla 1 en cuanto a la frecuencia del género y el tipo de discapacidad de los encuestados; en la Tabla 2 se observó la frecuencia de las alternativas de la Fuente interactiva de la resiliencia: "Yo soy"; en la tabla 3 se observó la frecuencia de las alternativas de la Fuente interactiva de la resiliencia: "Yo tengo" y en la tabla 4 se observó la frecuencia de las alternativas de la Fuente interactiva de la resiliencia: "Yo puedo", resaltando los datos relevantes y analizándolos de acuerdo a los estudios realizados por otros en situaciones similares y realidades con características afines.

Las interrogantes planteadas en la entrevista estructurada se le fueron formulando una a una a la directora del Departamento de Inclusión, Equidad social, la misma que fue respondiéndolas de forma oral y la información obtenida fue plasmada de forma textual en la guía de entrevista, con el fin de preservar sus contenidos, la fiabilidad y veracidad de la misma.

\section{Resultado}

Con el fin de determinar la resiliencia de los estudiantes con discapacidad de la Carrera de Trabajo Social UTM se aplicó la técnica de la encuesta, utilizando como instrumento un cuestionario, considerando los factores de la Escala de Resiliencia SV-RES, datos que se detallan a continuación:

\section{Tabla 1}

Sexo y tipo de discapacidad de los estudiantes de la Carrera de Trabajo social. U.T.M.

\begin{tabular}{lcc}
\hline Alternativas & Frecuencia & Porcentaje \\
\hline Género & 15 & $50 \%$ \\
Masculino & 15 & $50 \%$ \\
Femenino & 30 & $\mathbf{1 0 0 \%}$
\end{tabular}

\section{Tipo de discapacidad}




$\begin{array}{lcc}\text { Física } & 14 & 47 \% \\ \text { Intelectual } & 0 & 0 \% \\ \text { Motriz } & 0 & 0 \% \\ \text { Total } & 30 & 100 \%\end{array}$

Nota: Tomado de los estudiantes con discapacidad de la Carrera de Trabajo social. UTM. (2021).

De acuerdo a los resultados obtenidos en la encuesta aplicada a los estudiantes con discapacidad de la Carrera de Trabajo social de la Universidad Técnica de Manabí se pudo determinar que el $50 \%$ de los mismos son de sexo masculino y en la misma proporción, es decir 50 \%, corresponden al sexo femenino.

En cuanto a al tipo de discapacidad los resultados fueron los siguientes: la mayoría de los encuestados, representado en el 53,3 \% tienen discapacidad sensorial, lo que incluye a estudiantes con discapacidad auditiva, visual y de lenguaje; mientras que el $46,6 \%$ presentan discapacidad física, situaciones que no han sido una limitante para que los mismos hayan podido acceder a la educación superior y mantenerse acreditando cada nivel, puesto que los mismos estudian en diferentes niveles en lo que corresponde de primero a octavo.

\section{Tabla 2}

Frecuencia de la fuente interactiva de la resiliencia: "Yo soy":

\begin{tabular}{lcc}
\hline Alternativas & Frecuencia & Porcentaje \\
\hline Una persona fuerte & 0 & $0 \%$ \\
Capaz de controlar mi vida & 0 & $0 \%$ \\
Competente para alcanzar & 0 & $\%$ \\
metas & 17 & $57 \%$ \\
Orgullosos de mis logros & 13 & $43 \%$ \\
Seguro de mí mismo & 30 & $\mathbf{1 0 0 \%}$ \\
Total & 0 & \\
\hline
\end{tabular}

Nota: Tomado de los estudiantes con discapacidad de la Carrera de Trabajo social. UTM. (2021).

Al indicarle a los encuestados que elijan una fuente interactiva con la cuál ellos se identifican para definirse como "Yo soy", el 57 \% eligió la opción Orgulloso de mis logros, mientras que el 43 \% se inclinó por la alternativa seguro de sí mismo, considerándose que a pesar de las limitantes que puedan tener por su discapacidad, éstos se definen como personas capaces de afrontar las adversidades que se les pueden presentar debido a su discapacidad, las mismas que han sabido desarrollar considerando los estímulos de su entorno.

\section{Tabla 3}

Frecuencia de la fuente interactiva de la resiliencia: "Yo tengo": 


\begin{tabular}{lcc}
\hline Alternativas & Frecuencia & Porcentaje \\
\hline Confianza y optimismo & 0 & $0 \%$ \\
Facilidad para establecer contacto con nuevas personas & 0 & $0 \%$ \\
Amigos / familiares que me apoyan & 21 & $\mathbf{7 0 \%}$ \\
Compañeros que me apoyan en las actividades académicas & 8 & $\mathbf{2 7 \%}$ \\
Docentes que aplican metodología inclusiva & & $\mathbf{3 \%}$ \\
Total & 1 & $\mathbf{1 0 0 \%}$ \\
\hline
\end{tabular}

Nota: Tomado de los estudiantes con discapacidad de la Carrera de Trabajo social. UTM. (2021).

En relación a la fuente interactiva "Yo tengo" en la escala de Resiliencia SV-RES y en base a la encuesta realizada se determinó que: el $70 \%$ de los encuestados escogieron como factor indispensable para su resiliencia la ayuda por parte de amigos/familiares; el $27 \%$ consideran que son sus compañeros porque los apoyan en actividades académicas y el 3 \% que son los docentes debido a que aplican metodología inclusiva.

Los datos obtenidos permiten evidenciar que los estudiantes con discapacidad encuestados tienen en su entorno estímulos para desarrollar su resiliencia frente a las adversidades, tales como su familia, amistades, compañeros de clases y los docentes que en su mayoría llevan a cabo actividades de inclusión, lo que permite fortalecer la resiliencia de los implicados.

\section{Tabla 4}

Frecuencia de la fuente interactiva de la resiliencia: "Yo puedo":

\begin{tabular}{lcc}
\hline Alternativas & Frecuencia & Porcentaje \\
\hline Cumplir tareas asignadas & 0 & $0 \%$ \\
Planear actividades en base a metas & 0 & $0 \%$ \\
Trabajar en equipo & 23 & $\mathbf{7 7 \%}$ \\
Organizar mi tiempo y ser puntual & 0 & $0 \%$ \\
Motivar a otros & 7 & $\mathbf{2 3 \%}$ \\
Total & $\mathbf{3 0}$ & $\mathbf{1 0 0 \%}$ \\
\hline
\end{tabular}

Nota: Tomado de los estudiantes con discapacidad de la Carrera de Trabajo social. UTM. (2021).

En relación al factor "Yo puedo" se obtuvieron las siguientes respuestas: el 23 \% considera que puede trabajar en equipo, mientras que el $77 \%$ motiva a otros para alcanzar sus metas, lo que permite deducir 
que la mayoría de los estudiantes con discapacidad encuestados afirman que pueden llevar a cabo acciones para alcanzar metas y objetivos, por lo que se pueden consideran estudiantes resilientes en la educación superior.

En cuanto al enfoque cualitativo de la investigación, se realizó una entrevista a la Directora del DIESG de la UTM para determinar el objetivo, las propuestas, acciones y la percepción en cuanto a la resiliencia de los estudiantes registrados en dicha unidad. Se planteó la interrogante ¿Cuál es el objetivo del Departamento de Inclusión, Equidad Social y Género de la UTM ?, para lo cual Macias manifiesta que "EI Departamento de Inclusión Equidad Social y Género fue creado con el propósito de velar por el cumplimento de los derechos de los grupos de atención prioritaria, históricamente excluidos y aquellos que se encuentren en situación de vulnerabilidad" (Directora del Departamento de Inclusión, Equidad Social y Género de la UTM).

Con el fin de determinar las propuestas inclusivas que desarrolla el equipo del DIESG en beneficio de los estudiantes con discapacidad, se realiza la siguiente pregunta: ¿Cómo departamento de Inclusión social qué propuesta están prestos a ejecutar?, a lo que Macias manifiesta que como Directora y a la vez profesional en Trabajo social de esta Unidad proponen: crear y ejecutar estrategias para la inclusión y acompañamiento constante de docentes, empleados, trabajadores y estudiantes que se encuentren dentro del grupo de atención prioritaria o en situación de vulnerabilidad en la Universidad Técnica de Manabí, de igual forma concienciando y sensibilizando en temas de equidad, justicia, respeto, solidaridad a la comunidad universitaria, contribuyendo al buen vivir en la Universidad y la sociedad.

Es importante también identificar las acciones que actualmente está desarrollando el equipo multidisciplinario que conforma la Unidad en mención, para lo cual se planteó la siguiente interrogante: ¿Cuáles con las actividades que está desarrollando actualmente el Departamento de Inclusión y Equidad Social de Género con el fin de fomentar la inclusión?, razón por la cual Macias manifiesta que al ser un departamento integral, cuyo fin es eliminar las barreras que no permiten una inclusión pertinente de acuerdo a las necesidades educativas de cada estudiante con discapacidad, se ha trabajado coordinadamente con las autoridades rectoras de la U.T.M. en cuanto a la implementación de espacios arquitectónicos adecuados para el acceso a los predios universitarios y a los espacios de formación académica. Así mismo se está trabajando en el desarrollo de talleres educativos y de concienciación dirigidos a la comunidad educativa superior para fomentar la inclusión.

Por otra parte, se realizan actividades de capacitación dirigidas a los estudiantes con discapacidad y la orientación sobre la ruta a seguir para que éstos puedan acceder a los servicios que brinda la U.T.M. De igual forma se proporciona información sobre los requisitos y procesos para acceder a los programas del Ministerio de Inclusión, Económica y social y la Senescyt, para que los estudiantes puedan acceder a los 
programas que éstos ofertan. Una de las labores más relevantes que se desarrollan es la constante lucha por la inclusión de los estudiantes en cuanto a las actividades académicas, por lo que se trabaja en conjunto con autoridades y docentes para el logro de este fin.

Las personas con discapacidad que estudian en un centro de educación superior requieren de varios factores en su entorno para afrontar las adversidades que surgen en su proceso de formación académica, ya sean éstos familia, amigos, compañeros, docentes y autoridades de la comunidad educativa, así como también programas direccionados a brindar las condiciones adecuadas para su desarrollo académico, razón por la cual se le plantea la pregunta a la Directora del DIESG: ¿Considera usted que los estudiantes con discapacidad de la Carrera de Trabajo social son resilientes?, a lo que Macias manifiesta que los estudiantes con discapacidad de la Carrera de Trabajo Social son resilientes, debido al hecho de que éstos, a pesar de las dificultades y limitaciones que tienen, saben sobrellevar situaciones que en muchas ocasiones les impiden continuar con sus actividades académicas, ya sean éstos de tipo económico, material, emocional, de conocimiento, entre otros. (Directora del Departamento de Inclusión, Equidad Social y Género de la U.T.M.)

\section{Discusión}

La resiliencia es la capacidad que tienen las personas para encarar las situaciones adversas de la vida y los estudiantes con discapacidad que estudian en la carrera de Trabajo social de la Universidad Técnica de Manabí no son la excepción, quienes tienen actitudes idóneas para superar las situaciones negativas en el proceso, tal como lo mencionan (Zambrano, et al., 2018) considerando que:

Es de suma importancia que los estudiantes desarrollen prácticas actitudinales resilientes, ya que hay que reconocer que la resiliencia no es una capacidad que nace con el individuo, sino que puede aprenderse en cualquier momento de la vida, siempre y cuando haya en su alrededor otras personas que los impulse a ese logro. (p. 44 )

Las condiciones en las que se desenvuelven las personas con discapacidad son generalmente rodeadas de obstáculos o barreras para que puedan llevar una vida plena y adecuada, sin embargo, éstos han logrado adaptarse a las mismas, desarrollando condiciones adaptables para lograr un bienestar, puesto que existen estímulos en el entorno, los que a través de sus acciones fomentan la resiliencia, tal como lo manifiestan (Saavedra y Villalta, 2008):

En tal sentido, la resiliencia es un rasgo personal cultivado a lo largo de la historia del sujeto, y que posiblemente, se ha constituido como vinculo temprano siendo condición para el desarrollo de una particular apropiación de los sucesos de la vida. Es decir, aquello que el sujeto tiene como rasgo distintivo, ha sido aprendido en relación a otros. Siendo esto así, -aprendido- puede 
cambiar, o está en constante proceso de interpretación. Esto lleva al siguiente aspecto que los autores señalan como fuentes interactivas de la resiliencia. (p. 31)

La inclusión en los diferentes niveles educativos es un derecho adquirido por las personas con discapacidad, a lo que se debe dar estricto cumplimiento para el logro de la justicia social. En tanto que la Universidad Técnica de Manabí se suma a esta propuesta, coincidiendo con Segers et al. (2018) en cuanto al siguiente criterio:

La inclusión educativa surge como medida compensatoria dirigida a contrarrestar las inequidades en el acceso a servicios educativos a nivel mundial y de manera especial en los países en vías de desarrollo. La meta es incrementar la participación de los estudiantes en situaciones de vulnerabilidad en el aprendizaje, la cultura y las comunidades; y su meta es la eliminación de toda forma de exclusión. (p. 469)

Las metodologías inclusivas que aplican los docentes en la educación superior son indispensables para la formación integral del estudiante con discapacidad, pues de ésta depende el desarrollo de la capacidad resiliente. Es así que Cobeñas (2020) plantea que:

Las personas con discapacidad son quienes tienen derecho a que las escuelas se transformen en pos de una educación inclusiva, y no las escuelas las que pueden decidir a qué alumnos educar. Siguiendo las demandas del movimiento de personas con discapacidad, así como los aportes de los estudios sociales de la discapacidad, se parte de la convicción de que el pleno ejercicio del derecho a la educación de los grupos excluidos del sistema escolar, o segregados en instituciones "especiales", sólo puede darse por medio de una educación inclusiva que problematice esta categorización y tenga como objetivo una educación para todos y todas. (p. 66)

\section{Conclusión}

Las personas con discapacidad son aquellas que tienen ciertas limitantes para desarrollar todas las actividades en su diario vivir; sin embargo, se aplican leyes y normativas dirigidas hacia su inclusión en diferentes ámbitos. En cuanto a la educación superior la inclusión de las personas con discapacidad es una realidad para lo cual, los implicados desarrollan la resiliencia como capacidad para afrontar las adversidades junto a entornos positivos conformados por la familia, amistades, compañeros, docentes, entre otros.

Los estudiantes sujetos de estudio de la carrera de Trabajo Social tienen diferentes tipos de discapacidad, entre las cuales se mencionan la sensorial, lo que incluye discapacidad auditiva, visual y de lenguaje, que 
la padecen en su mayoría, así mismo otros tienen discapacidad física; éstos cursan en diferentes niveles de primero a octavo y corresponden al sexo masculino y femenino en iguales proporciones.

Los estudiantes con discapacidad de la Carrera de Trabajo Social de la Universidad Técnica de Manabí se consideran resilientes, puesto que tienen capacidades para afrontan adversidades, debido a que la mayoría afirma identificarse con las fuentes interactivas de resiliencia "yo soy, yo tengo y yo puedo" consideradas en las dimensiones de la escala validada de SV-RES, para lo cual cuentan con el apoyo de personas que constituyen su entorno como la familia, amigos, compañeros, docentes, Unidad de inclusión, Universidad Técnica de Manabí y Programas de bienestar del Estado ecuatoriano.

\section{Referencias}

Cobeñas, P. (2020). Exclusión Educativa de Personas con Discapacidad: Un problema pedagógico. REICE. Revista Iberoamericana sobre Calidad, Eficacia y Cambio en Educación 18(1), 65-81. https://doi.org/10.15366/reice2020.18.1.004

Consejo Nacional para la Igualdad de Discapaciddes (2008) Manual de Atención en Derechos de Personas con Discapacidad en la Función Judicial. Ecuador. http://www.funcionjudicial.gob.ec/www/pdf/Manual-atencion-discapacidades.pdf

Constitucion de la Republica del Ecuador. [Const], 20 de octubre de 2008. https://educacion.gob.ec/wpcontent/uploads/downloads/2012/08/Constitucion.pdf

Decreto Ejecutivo 194. Reglamento a la Ley Orgánica de Discapacidades. (2017, 27 de octubre). https://www.consejodiscapacidades.gob.ec/wpcontent/uploads/downloads/2017/11/Regalamento-lod-decre_-194.pdf

Ley N. ${ }^{\circ}$ 796, Ley Orgánica de Discapacidades. (26 de junio de 2012). https://www.consejodiscapacidades.gob.ec/wp-content/uploads/downloads/2016/06/LeyOrganica-de-Discapacidades.pdf

Martinez, R. (2017). Resiliencia en jóvenes con discapacidad ¿difiere según la tipología y etapa en la que se adquiere la discapacidad?. RUA, Universidad de Alicante. Boletín de Psicología. (105) , 75-89. https://rua.ua.es/dspace/bitstream/10045/25976/1/N105-5.pdf

Moscoso-Escalante, S. y Castañeda-Chang, A. (2018) Validez y confiabilidad de la Escala de Resiliencia (SV-RES) para adultos mayores en Lima metropolitana. Búsqueda, 5(21), 165-181. https://doi.org/10.21892/01239813.419

Organización Mundial de la Salud. (2011). Informe mundial sobre la discapacidad. Observatorio Discapacidad Física. https://bibliotecadigital.indh.cl/handle/123456789/302 
Ormaza-Cevallos, G., Cevallos-Caicedo, C., Lozano-Jaramillo, G. y Pico-Macias, M. (2018). Resiliencia desde la teoría. En Macias, A. (ed.), Resiliencia: Experiencias investigativas y prospectivas (pp. 6988). Editorial 3Ciencias.

Romero, S., Alava, L., Alcivar, E. y Moreira, T. (2018). Resiliencia y Trabajo social. En Macias, A. (ed.), Resiliencia: Experiencias investigativas y prospectivas (pp. 69-88). Editorial 3Ciencias.

Saavedra-Guajardo, E. y Villalta-Paucar, M. (2008). Medición de las características resilientes, un estudio Comparativo en personas entre 15 y 65 años. Liberabit, 14(14), 32- 40. http://www.scielo.org.pe/scielo.php?script=sci_arttext\&pid=S172948272008000100005\&lng=e s\&tIng=es.

Salvo-Garrido, S., Gálvez-Nieto, J. y Martin-Parihuén, S. (2021). Resiliencia académica: Comprendiendo las relaciones familiares que la promueven. Revista Electrónica Educare (Educare Electronic Journal, 25(2), 1-18. https://doi.org/10.15359/ree.25-2.20

Segers, D., Bravo, S. y Villafuerte, J. (2018). La resiliencia y estilos de afrontamiento de jóvenes estudiantes con discapacidad. Revista de Curriculum y Formación del Profesorado, 22(4) 463487. https://orcid.org/0000-0003-3639-2678

Secretaria de Educacion superior Ciencia y Tecnologia. (2014). Informe sobre la Accesibilidad Universal en Ecuador. https://www.educacionsuperior.gob.ec/acceso-a-la-educacion-superior-presenteen-las-jornadas-de-inclusion-hacia-la-accesibilidad-universal-en-el-ecuador/

Secretaria de Educacion superior Ciencia y Tecnologia. (2019, 11 de abril). Boletín de prensa № 083, Senescyt plantea una educación superior en igualdad de oportunidades. https://n9.cl/0n9kz

Serrano-Nortes, E., Gómez Díaz M. y Reche-García C. (2021). Resiliencia y optimismo en gimnasia rítmica. Retos, 41, 581-588. https://doi.org/10.47197/retos.v0i41.83086

Tarazona-Meza, A., Saldarriaga-Villamil, K., Vazquez-Perez, A., Pinargote-Macias, E. y DominguezUtreras, L. (2018). Cuatro aristas de la resiliencia en Manabí Ecuador. En Macias, A. (ed.), Resiliencia: Experiencias investigativas y prospectivas (pp. 69-88). Editorial 3Ciencias.

Universidad Técnica de Manabí. (2021). Misión, Visión y Valores. https://www.utm.edu.ec/launiversidad/nuestra-universidad/mision-vision-y-valores

Universidad Técnica de Manabí. (2019). Reglamento de Políticas de Acción Afirmativa. https://www.utm.edu.ec/la-universidad/nuestra-universidad/reglamentos

Universidad Técnica de Manabí. (2012). Departamento de Inclusión, Equidad Social y Género. https://www.utm.edu.ec/inclusion/ 
Verdugo, M. (2003). Aportaciones de la definición de retraso mental (AAMR, 2002) a la corriente inclusiva de las personas con discapacidad. Salamanca Amarú.

https://sid.usal.es/idocs/f8/fdo6569/verdugo.pdf

Zambrano-Santos, R., Arteaga-Muñoz, K., Ubillus-Santos, S., Sanchez-Rodriguez, J y Macias-Alvia, A. (2018). La resiliencia en los estudiantes universitarios. En Macias, A. (ed.), Resiliencia: Experiencias investigativas y prospectivas (pp. 69-88). Editorial 3Ciencias.

\section{Contribución de los autores}

TKMC: Metodología, investigación, redacción: borrador original.

EMVG: Conceptualización, análisis formal, investigación, redacción: borrador original.

SARC: Metodología, supervición, validación, redacción: revisión y edición.

\section{Fuentes de financiamiento.}

La investigación fue realizada con recursos propios.

\section{Conflictos de interés}

Las autoras de este artículo manifiestan no tener ningún conflicto de intereses

\section{Correspondencia}

tatiana.moreira@ild.ec 